\title{
Analysis of Initial Clinical and CT Features of COVID-19 Pneumonia in Different Clinical Types
}

Wei Li

The Fifth Affiliated Hospital, Sun Yat-sen University https://orcid.org/0000-0003-0236-4214

\section{Wenjun Yu}

Jianwei Liao

Lin Yao

Yijie Fang

Cunxue Pan

Qing Xie

Huaqian Cui

Xiang Zeng

Shaolin Li ( $\sim$ lishlin5@mail.sysu.edu.cn )

Department of Radiology, The Fifth Affiliated Hospital, Sun Yat-sen University https://orcid.org/00000003-1965-0217

\section{Research Article}

Keywords: COVID-19; coronavirus infections; viral pneumonia, computed tomography; clinical types

Posted Date: May 21st, 2020

DOI: https://doi.org/10.21203/rs.3.rs-30395/v1

License: (c) (1) This work is licensed under a Creative Commons Attribution 4.0 International License. Read Full License 


\section{Abstract}

Background Different clinical classifications of COVID-19 pneumonia patients have different clinical and CT features, which is very important for the treatment after admission. As the epidemic situation in China continues to improve, it is particularly important to re-clarify the correlation between them.

Methods 97 confirmed patients with COVID-19 pneumonia were enrolled from January 17, 2019 to February 21,2020 , including 75 mild/ordinary cases and 22 severe/critical cases. The clinical data and initial chest CT images of the patients were reviewed and compared. The risk factors associated with disease severity were analyzed.

Results Compared with the mild/ordinary patients, the severe/critical patients had older ages, higher incidence of comorbidities, first CT positive, CT always negative and fever. Mild/ordinary patients had lower body temperature than mild/ordinary patients. The incidences of large/multiple GGO in severe/critical patients were significantly higher than those of the mild/ordinary patients, furthermore, severe/critical patients showed higher incidences of 4-5 lobe infections than the ordinary patients. The CT scores of severe/critical patients were significantly higher than those of the ordinary patients $(P<$ 0.001). The clinical factors of age, sex, comorbidities, hypertension, diabetes mellitus, heart disease, pharyngeal discomfort, abdominal pain/diarrhea, temperature and CT score were risk factors for severe/critical COVID-19 pneumonia.

Conclusion The initial clinical and CT characteristics have certain significance for the clinical classification of COVID-19 respiratory infection. Especially in terms of CT score, it can predict the trend of clinical classification of patients to a certain extent.

\section{Introduction}

Since 31 December 2019, many cases of an "unknown viral pneumonia" have been found in Wuhan, China[1]. On February 12, 2020, the World Health Organization (WHO) announced that the official name of the disease caused by the novel coronavirus is COVID-19[2]. Over time, viral infections have been reported all over the world and the infection has spread rapidly in worldwide[3-7]. As of May 10, 2020, a total of 3,855,788 cases of COVID-19 have been confirmed in more than 200 countries and regions, and this number is still rising[8]. Since this viral infection has developed into a global emergency, the diagnosis of infected patients is particularly important. Although the source of the virus has not been fully clarified so far, the diagnosis of COVID-19 still mainly depends on the results of reverse transcriptase polymerase chain reaction (RT-PCR) detection, which possesses a high specificity but a poor sensitivity[9]. And with the increase in the number of patients, its testing efficiency will be greatly reduced. As more and more people with asymptomatic infections appear, the diagnosis becomes more and more difficult. According to some recent on-line publication, some of the patients were diagnosed as COVID-19 without fever or other discomforts in the early stage, and even with negative nucleic acid tests at first few 
times[10]. Therefore, combining clinical findings with imaging features can help make an early diagnosis of COVID-19.

Chest CT scan is one of the important methods for the diagnosis of lung infection. The typical radiographic imaging feature of COVID-19 pneumonia is ground-glass patches (GGO), and there can be some other changes like consolidation, reticulation /interlobular septal thickening, irregular solid nodules and fibrous stripes[11, 12]. However, previous reports are only a general retrospective description without analysis of different clinical types[13, 14]. Although there have been previous studies of semi-quantitative CT scores[15, 16], they only used the range of lesions to score and there is no combination of range and density assessment methods for CT scoring which will cause the loss of some useful information. Moreover, most of the previous risk factor analysis was single factor analysis[15], with some confounding factors in it, which could not really reflect the risk factors of the disease. Therefore, 97 patients admitted in our hospital are classified according to different clinical types, and their clinical symptoms and pulmonary CT imaging features were analyzed and summarized, in order to explore the related factors of severe /critical patients and further improve the early diagnosis of the disease.

\section{Methods}

Our ethics committee approved this retrospective study, and the requirement for informed consent was waived. because this study would not cause damage to the patient's body.

\section{Patients and Clinical Characteristics}

98 confirmed patients with COVID-19 pneumonia in our hospital were collected in this study from January 17, 2019 to February 21, 2020. The inclusion criteria were as follows: A) having an epidemiological history; B) real-time RT-PCR detection of SARS-CoV-2 nucleic acid positive in throat swabs; $\mathrm{C}$ ) having had at least one chest CT scan during hospitalization. The mild/ordinary patients showed or did not show the corresponding clinical symptoms and lung imaging findings. The severe/critical patients met any of the following condition:1) respiratory rate $\geq 30$ breaths per minute; 2 ) finger of oxygen saturation $\leq 93 \%$ in a resting state; 3 ) arteria oxygen tension ( $\mathrm{PaO2}$ )/inspiratory oxygen fraction (FiO2) $\leq 300 \mathrm{mmHg}(1 \mathrm{mmHg}=0.133 \mathrm{kPa}) ; 4)$ pulmonary imaging showed that the lesions significantly progressed $>50 \%$ within $24-28$ hours 5 ) respiratory failure occurred and mechanical ventilation required; 6 ) shock occurred; 7) Combining other organ failure and required ICU monitoring treatment. The exclusion criteria were COVID-19 patients without CT scan. Finally, 1 patient was excluded due to no CT scan and 97 patients were included. The clinical parameters were collected and evaluated, including gender, age, age groups, cluster onset, comorbidities (hypertension, diabetes mellitus, heart disease, old tuberculosis, cancers), symptoms (fever, cough and expectoration, pharyngeal discomfort, myalgia, fatigue, dizziness and headache, chest tightness, abdominal pain/diarrhea) and temperature.

\section{CT Image Acquisition}


Due to the large number of patients detected., we used 3 different CTs for chest examination in order to avoid cross-infection. All patients underwent at least one non-enhanced CT examination. The model and parameters of the machine are as follows: A) PET-CT uMI780, 120KV, automatic mAs, layer thickness $1.0 \mathrm{~cm}$, layer spacing $1.0 \mathrm{~cm} ; \mathrm{B}) \mathrm{uCT} 760,120 \mathrm{KV}$, automatic $\mathrm{mAs}$, layer thickness $1.0 \mathrm{~cm}$, layer spacing $1.0 \mathrm{~cm}$;C) KAIPU CT precision $32,120 \mathrm{KV}$, automatic $\mathrm{mAs}$, layer thickness $1.1 \mathrm{~cm}$, layer spacing $0.7 \mathrm{~cm}$. Two chest radiologists with 10 and 7 years of experience independently evaluated the CT images in consensus while they were blinded to the names and clinical data of these patients. For each of the 97 patients, the initial chest CT findings were evaluated for the following characteristics: patchy/punctate GGO, large/multiple GGO, consolidation, reticulation/interlobular septal thickening, irregular solid nodules, fibrous stripes and no related lesions. Patchy/punctate GGO is defined as a single ground glass lesion less than $5 \mathrm{~cm}$ in diameter of each lobe, and large/multiple GGO is defined as a single lesion larger than 5 $\mathrm{cm}$ in diameter or multiple lesions in each lobe.

\section{Image Interpretation and Scoring}

To semi-quantitatively analyze the severity of the lesion, two chest radiologists with 10 and 7 years of experience independently scored the $\mathrm{CT}$ images of each patient. The inter-observer and intra-observer agreement of CT score measurement was analyzed by interclass correlation coefficient (ICC). Each lobe is scored separately based on range and density. The range scoring rules of each lobe are as follows: score $0,0 \%$ involvement; score $1,1 \%$ to $25 \%$ involvement; score $2,26 \%$ to $50 \%$ involvement; score $3,51 \%$ to $75 \%$ involvement; score $4,75 \%$ to $100 \%$ involvement. The density score is based on the average CT value of the lesion and is defined as follows: score 0 , no lesions; score 1, CT value between $-799 \mathrm{HU}$ and $-600 \mathrm{HU}$; score 2, CT value between $-599 \mathrm{HU}$ and $-400 \mathrm{HU}$; score 3, CT value between $-399 \mathrm{HU}$ and $-200 \mathrm{HU}$; score 4, CT value between $-199 \mathrm{HU}$ and $\mathrm{OHU}$. The average CT value of each lung lobe is the average of the maximum and minimum values. The final total CT score obtained is the sum of range score and density score. Each lobe has a final score between $0-8$, so the final possible score is between $0-40$. The final score is averaged between the results of the two observers.

\section{Statistical Analysis}

All statistical analyses were performed by using SPSS statistical software (version20.0, IBM). Categorical variables were described as frequency rates and percentages, and quantitative variables were described using mean or median values. In univariate analysis, the chi-square test and Fisher exact test were used for categorical variables. Quantitative variables were tested for normality using levene test. Normally distributed data were analyzed by independent sample $t$ test, otherwise, the ranks test. In multivariate analysis, the statistical model was constructed using binary logistic regression. For logistic regression analysis, quantitative variables were transformed into categorical variables. P values $<0.05$ were considered as statistically significant.

\section{Results}


Clinical characteristics in 97 patients on admission are summarized in Table 1. In these patients, the average age of severe/critical group is greater than that of the mild/ordinary group (mean age, 58.2 years $[S D, 13.2]$ vs 41.1 years [SD,17.4]; $P<0.001$ ), and there are also statistical differences between different age groups. Most of the two groups of patients are clustered onset. The severe/critical group had more comorbidities of hypertension, diabetes mellitus and old tuberculosis and had a higher probability of positive initial CT compared with the mild/ordinary group. It is worth noting that all severe/critical patients had a positive rate of $100 \%$ on continuous CT examination. The most common symptoms in these patients were fever (55/97,56.7\%) and cough/expectoration (43/97,44.3\%). Less common symptoms were pharyngeal discomfort, myalgia, fatigue, dizziness/ headache, chest tightness and abdominal pain/diarrhea. But only the fever between the two groups has a statistically significant difference $(P=0.030)$.

The body temperature of the severe/critical group is higher than that of the mild/ordinary group (mean temperature, $38.3^{\circ} \mathrm{C}[\mathrm{SD}, 0.9]$ vs $37.6^{\circ} \mathrm{C}[\mathrm{SD}, 0.9] ; \mathrm{P}=0.001$ ) and this is also statistically different in the temperature grouping.

\section{Chest CT findings of the initial CT}

The most common chest CT features of both groups included patchy/ punctate GGO $(65 / 97,67.0 \%)$ and large/multiple GGO (56/97,57.5\%). Other relatively uncommon features are consolidation, reticulation /interlobular septal thickening, irregular solid nodules and fibrous stripes (Table 2). The occurrence rates of large/multiple GGO in severe/critical patients were significantly higher than those of the mild/ordinary patients $(P=0.013)$. The lesions in both groups are common in the subpleural distribution. The numbers and distribution of lung lobes affected were also statistical differences between the two groups. The severe/critical group had more lung lobes involved than mild/ordinary group $(P<0.001)$ and the former was more likely to involve bilateral lung lobes at the same time $(P=0.026)$. Figure 1 showed the CT appearance of a ordinary patient.

\section{CT score findings}

The CT scores of the severe/critical patients were significantly higher than those of the mild/ordinary patients $(P<0.001)$. The average score for severe/critical group is 18.8 , but for the mild group was 8.13(Table 3). And when the CT score were divided into 4 groups on average, there are still significant statistical differences between the groups. In severe/critical group, the proportion of patients with CT scores above 20 is very high.

\section{Multivariate analysis outcomes}

The variables selected to construct binary logistic regression include demographic characteristics (age, sex, cluster onset), past medical history (hypertension, diabetes mellitus, heart disease, old tuberculosis, cancers), symptoms (temperature, cough and expectoration, pharyngeal discomfort, myalgia, fatigue, dizziness and headache, chest tightness, abdominal pain / diarrhea, comorbidities, consolidation) and CT 
score. Table 4 showed the results of multivariate analysis for different clinical types of COVID-19 Pneumonia. The factors of age, gender, comorbidities, hypertension, diabetes mellitus, heart disease, pharyngeal discomfort, abdominal pain/diarrhea, temperature and CT score were risk factors for severe/critical COVID-19 pneumonia.

\section{Discussion}

Although there are certain similarities between the severe/critical patients and mild/ordinary patients, they still have differences in some clinical and imaging characteristics. In this study, there is no obvious correlation between the incidence of pneumonia and gender, which means that the probability of pneumonia in men and women is equal. We found that the elder patients are the majority of severe/critical group, with a higher proportion of underlying diseases like hypertension, diabetes mellitus and old tuberculosis. This may be related to poor physical resistance and low immunity of the elderly patients[17]. Compared with the mild/ordinary group, the severe/critical group had higher incidences of fever, but there was no difference in other symptoms like cough and expectoration, pharyngeal discomfort, myalgia, fatigue, dizziness/ headache, chest tightness and abdominal pain/diarrhea. The average body temperature of patients in the severe/critical group is higher than that in the mild/ordinary group, which may indicate that the immune system of severe/critical patients were highly activated. The occurrence of these symptoms and signs have an effective hint in clinical diagnosis and classification.

Chest CT scan can accurately assess the distribution and density of inflammation in the lung lobes[18]. In this study, the most common chest CT features of both groups are patchy/ punctate GGO and large/multiple GGO. In other words, GGO was the most common imaging manifestation of new coronary pneumonia, which suggests that the COVID-19 pneumonia is mainly based on the exudation of interstitial lung. This also means that the pathological mechanism of the disease is dilatation and congestion of alveolar septal capillaries, fluid exudation in the alveolar cavity and interstitial edema of the leaflet septum[19,20]. We divided the type of GGO into patchy/ punctate GGO and large/multiple GGO, and found that large GGO accounted for a large proportion of critically ill patients. This means that when the lung CT of the patient indicates an increase in GGO or GGO develops into a large area, the doctor should be alert to the possibility of developing into a severe/critical type. Other CT features like consolidation, reticulation /interlobular septal thickening, irregular solid nodules and fibrous stripes are not statistically significant, although some of them have a $\mathrm{P}$ value close to 0.05 . One possible reason is related to the small sample size. But we still observe that in severe/critical patients, the probability of CT negative is very low. The numbers and position of lung lobes affected were also statistical differences between the two groups. In severe/critical patients, the number of lung lobes involved and the probability of involving bilateral lung lobes is greater. This suggests that the lesions of severe/critical patients are more likely to spread to each lung lobe.

The CT scores of the severe/critical group were significantly higher than those of the ordinary group $(P<0.001)$, which means that the CT score can accurately distinguish between two clinical types. If a patient's CT score is higher than 20 , the doctors need to be alert to the possibility that the patient will 
develop into severe/critical type of COVID-19 pneumonia. This is of great importance for subsequent treatment and follow-up.

In multi-factor analysis, our research found some risk factors related to COVID-19 pneumonia, such as age, gender, comorbidities, hypertension, diabetes mellitus, heart disease, pharyngeal discomfort, abdominal pain/diarrhea, temperature and CT score. The OR interval of some variables is too wide because of the low incidence of certain variables (such as diabetic brain, hypertension) in our study. Therefore, in the multi-factor analysis, there will be a large confidence interval of the or value. To reduce this situation, the sample size will need to be increased.

In conclusion, correctly distinguishing the clinical classification is of great significance to diagnosis and treatment, which is also related to the recovery of patients. Therefore, in the usual diagnosis and treatment work, we should pay attention to the importance of patients with pneumonia. Among the diagnosed patients, the clinical and imaging methods are suitable for clinical classification. Especially in the case of asymptomatic infection, CT score is an effective classification method.

\section{Declarations}

Competing interests: The authors declare no competing interests.

\section{References}

1. Novel Coronavirus - China. In: Who.int Web site. Who.int Web site. https://www.who.int/csr/don/12january-2020-novel-coronavirus-china/en/. Accessed May 82020.

2. Novel Coronavirus(2019-nCoV) Situation Report - 22. Who.int Web site. https://www.who.int/emergencies/diseases/novel-coronavirus-2019/situation-reports/. Accessed May 102020.

3. Mousavi SH, Shah J, Giang HTN, Al-Ahdal TMA, Zahid SU, Temory F et al. The first COVID-19 case in Afghanistan acquired from Iran. Lancet Infect Dis. 2020. doi:10.1016/S1473-3099(20)30231-0.

4. Tuite AR, Ng V, Rees E, Fisman D. Estimation of COVID-19 outbreak size in Italy. Lancet Infect Dis. 2020. doi:10.1016/S1473-3099(20)30227-9.

5. Verity R, Okell LC, Dorigatti I, Winskill P, Whittaker C, Imai N et al. Estimates of the severity of coronavirus disease 2019: a model-based analysis. Lancet Infect Dis. 2020. doi:10.1016/S14733099(20)30243-7.

6. Xiong N, Wang T, Lin Z. Invisible spread of SARS-CoV-2. Lancet Infect Dis. 2020. doi:10.1016/S14733099(20)30263-2.

7. Yan G, Lee CK, Lam LTM, Yan B, Chua YX, Lim AYN et al. Covert COVID-19 and false-positive dengue serology in Singapore. Lancet Infect Dis. 2020. doi:10.1016/S1473-3099(20)30158-4.

8. Coronavirus disease 2019 (COVID-19) Situation Report -110. Who.int Web site. https://www.who.int/emergencies/diseases/novel-coronavirus-2019/situation-reports/. Accessed 
May 102020.

9. Ai T, Yang Z, Hou H, Zhan C, Chen C, Lv W et al. Correlation of Chest CT and RT-PCR Testing in Coronavirus Disease 2019 (COVID-19) in China: A Report of 1014 Cases. Radiology. 2020:200642. doi:10.1148/radiol.2020200642.

10. He G, Sun W, Fang P, Huang J, Gamber M, Cai J et al. The clinical feature of silent infections of novel coronavirus infection (COVID-19) in Wenzhou. J Med Virol. 2020. doi:10.1002/jmv.25861.

11. Chung M, Bernheim A, Mei X, Zhang N, Huang M, Zeng X et al. CT Imaging Features of 2019 Novel Coronavirus (2019-nCoV). Radiology. 2020;295(1):202-7. doi:10.1148/radiol.2020200230.

12. Zhu T, Wang Y, Zhou S, Zhang N, Xia L. A Comparative Study of Chest Computed Tomography Features in Young and Older Adults With Corona Virus Disease (COVID-19). J Thorac Imaging. 2020. doi:10.1097/RTI.0000000000000513.

13. Han X, Cao Y, Jiang N, Chen Y, Alwalid O, Zhang X et al. Novel Coronavirus Pneumonia (COVID-19) Progression Course in 17 Discharged Patients: Comparison of Clinical and Thin-Section CT Features During Recovery. Clin Infect Dis. 2020. doi:10.1093/cid/ciaa271.

14. Pan Y, Guan H. Imaging changes in patients with 2019-nCov. Eur Radiol. 2020. doi:10.1007/s00330020-06713-Z.

15. Li K, Wu J, Wu F, Guo D, Chen L, Fang Z et al. The Clinical and Chest CT Features Associated with Severe and Critical COVID-19 Pneumonia. Invest Radiol. 2020. doi:10.1097/RLI.0000000000000672.

16. Zhou Z, Guo D, Li C, Fang Z, Chen L, Yang R et al. Coronavirus disease 2019: initial chest CT findings. Eur Radiol. 2020. doi:10.1007/s00330-020-06816-7.

17. Shi H, Han X, Jiang N, Cao Y, Alwalid O, Gu J et al. Radiological findings from 81 patients with COVID19 pneumonia in Wuhan, China: a descriptive study. Lancet Infect Dis. 2020. doi:10.1016/S14733099(20)30086-4.

18. Zhu Y, Liu YL, Li ZP, Kuang JY, Li XM, Yang YY et al. Clinical and CT imaging features of 2019 novel coronavirus disease (COVID-19). J Infect. 2020. doi:10.1016/j.jinf.2020.02.022.

19. Notice on Printing and Distributing the New Coronary Virus Pneumonia Diagnosis and Treatment Plan (Trial Version 7). nhc.gov.cn Web site. http://www.nhc.gov.cn/yzygj/new_index.shtml. Accessed March 192020.

20. Xu Z, Shi L, Wang Y, Zhang J, Huang L, Zhang C et al. Pathological findings of COVID-19 associated with acute respiratory distress syndrome. Lancet Respir Med. 2020;8(4):420-2. doi:10.1016/S22132600(20)30076-X.

\section{Tables}

Table 10Univariate analysis for clinical characteristics of different clinical types of COVID-19 Pneumonia 


\begin{tabular}{|c|c|c|c|c|}
\hline Variables & Total $(n=97)$ & Mild/Ordinary Group $[n=75 \square$ & Severe/critical Group $(\mathrm{n}=22)$ & $P$ Value \\
\hline Sex & & & & 0.174 \\
\hline Male & $43(46.4 \%)$ & $43(57.3 \%)$ & $13(59.1 \%)$ & \\
\hline Female & $52(53.6 \%)$ & $32(42.7 \%)$ & $9(40.9 \%)$ & \\
\hline Age & $45.0(18.0)$ & $41.1(17.4)$ & $58.2(13.2)$ & $<0.001$ \\
\hline Age group & & & & $<0.001$ \\
\hline Junior (0-17) & $6(6.2 \%)$ & $6(8.0 \%)$ & $0(0)$ & \\
\hline Youth (18-44) & $44(45.4 \%)$ & $39(52.0 \%)$ & $5(22.7 \%)$ & \\
\hline Middle age (45-59) & $23(23.7 \%)$ & $20(26.7 \%)$ & $3(13.6 \%)$ & \\
\hline Senior $(\geqq 60)$ & $24 \square 24.7 \% \square$ & $10(13.3 \%)$ & $14(63.6 \%)$ & \\
\hline Cluster onset & $83(85.6 \%)$ & $65(86.7 \%)$ & $18(81.8 \%)$ & 0.730 \\
\hline Comorbidities & $21(21.6 \%)$ & $11 \square 14.7 \% \square$ & $10(45.5 \%)$ & 0.006 \\
\hline Hypertension & $14(14.4 \%)$ & $7(9.3 \%)$ & $7(31.8 \%)$ & 0.015 \\
\hline Diabetes mellitus & $5(5.2 \%)$ & $1(1.3 \%)$ & $4(18.2 \%)$ & 0.009 \\
\hline Heart disease & $4(4.1 \%)$ & $2(2.7)$ & $2(9.1 \%)$ & 0.220 \\
\hline Old tuberculosis & $2(2.1 \%)$ & $0(0)$ & $2(9.1)$ & 0.050 \\
\hline Cancers & $3(3.1 \%)$ & $2(2.7 \%)$ & $1(4.5 \%)$ & 0.542 \\
\hline First RT-PCR positive & $67(69.1 \%)$ & $50(66.7 \%)$ & $17(77.3 \%)$ & 0.344 \\
\hline First CT positive & $72(74.2 \%)$ & $52(69.3 \%)$ & $20(90.9 \%)$ & 0.042 \\
\hline $\mathrm{CT}$ always negative & $18(18.6 \%)$ & $18(24.0 \%)$ & $0(0)$ & 0.010 \\
\hline No symptoms & $12(12.4 \%)$ & $11(14.7 \%)$ & $1(4.5 \%)$ & 0.287 \\
\hline Fever & $55(56.7 \%)$ & $38(50.7 \%)$ & $17(77.3 \%)$ & 0.030 \\
\hline Cough and expectoration & $43(44.3 \%)$ & $32(42.7 \%)$ & $11(50.0 \%)$ & 0.628 \\
\hline Pharyngeal discomfort & $13(13.4 \%)$ & $8(10.7 \%)$ & $5(22.7 \%)$ & 0.163 \\
\hline Myalgia & $6(6.2 \%)$ & $3(4.0 \%)$ & $3(13.6 \%)$ & 0.128 \\
\hline Fatigue & $8(8.2 \%)$ & $6(8.0 \%)$ & $2(9.1 \%)$ & 1.000 \\
\hline Dizziness and headache & $7(7.2 \%)$ & $5(6.7 \%)$ & $2(9.1 \%)$ & 0.655 \\
\hline Chest tightness & $4(4.1 \%)$ & $4(5.3 \%)$ & $0(0)$ & 0.571 \\
\hline Abdominal pain/Diarrhea & $6(6.2 \%)$ & $3(4.0 \%)$ & $3(13.6 \%)$ & 0.128 \\
\hline Temperature $\left({ }^{\circ} \mathrm{C}\right)$ & $37.7(0.9)$ & $37.6(0.8)$ & $38.3(0.9)$ & 0.001 \\
\hline Temperature group & & & & $<0.001$ \\
\hline Low fever $\left(37.8-38.0^{\circ} \mathrm{C}\right)$ & $78(70.4 \%)$ & $66(88.0 \%)$ & $12(54.5 \%)$ & \\
\hline Moderate fever $\left(38.1-39.0^{\circ} \mathrm{C}\right)$ & $17(17.5 \%)$ & $8(10.7 \%)$ & $9(40.9 \%)$ & \\
\hline High fever $\left(39.1-41.0^{\circ} \mathrm{C}\right)$ & $2(2.1 \%)$ & $1(1.3 \%)$ & $1(4.5 \%)$ & \\
\hline
\end{tabular}

Data are n (\%), mean (SD)

Table 2₫CT Features between mild/ordinary group and severe/critical group

\begin{tabular}{|c|c|c|c|c|}
\hline Variables & Total(n=97) & Mild/Ordinary Group $\square n=75 \square$ & Severe/critical Group(n=22) & $P$ Value \\
\hline Patchy/ Punctate GGO & $65(67.0 \%)$ & $48(64.0 \%)$ & $17(77.3 \%)$ & 0.308 \\
\hline Large/Multiple GGO & $56(57.5 \%)$ & $38(50.7 \%)$ & $18(81.8 \%)$ & 0.013 \\
\hline Consolidation & $14(14.4 \%)$ & $8(10.7 \%)$ & $6(27.3 \%)$ & 0.080 \\
\hline Reticulation / Interlobular septal thickening & $13(13.4 \%)$ & $8(10.7 \%)$ & $5(22.7 \%)$ & 0.163 \\
\hline Irregular solid nodules & $6(6.2 \%)$ & $6(8.0 \%)$ & $0(0)$ & 0.322 \\
\hline Fibrous stripes & $8(8.2 \%)$ & $4(5.3 \%)$ & $4(18.2 \%)$ & 0.075 \\
\hline None lesions & $25(25.8 \%)$ & $23(30.7 \%)$ & $2(9.1 \%)$ & 0.042 \\
\hline Numbers of Lobes Affected & & & & $<0.001$ \\
\hline 0 Lobes & $26 \square 26.8 \% \square$ & $24 \square 32.0 \% \square$ & $2 \square 9.1 \% \square$ & \\
\hline $1 \square 3$ Lobes & $38 \square 39.2 \% \square$ & $34 \llbracket 45.3 \% \square$ & $4 \square 18.2 \% \square$ & \\
\hline $4 \llbracket 5$ Lobes & $33 \square 34.0 \% \square$ & $17 \square 22.7 \% \square$ & $16 \square 72.7 \% \square$ & \\
\hline Distribution of LobesAffected & & & & 0.026 \\
\hline No position & $26(26.8 \%)$ & $24(32.0 \%)$ & $2(9.1 \%)$ & \\
\hline Only left & $12(12.4 \%)$ & $11(14.7 \%)$ & $1(4.5 \%)$ & \\
\hline Only right & $7(7.2 \%)$ & $6(8.0 \% \square$ & $1(4.5 \%)$ & \\
\hline Bilateral lung disease & $52(53.6 \%)$ & $34(45.3 \%)$ & $18(81.8 \%)$ & \\
\hline Subpleural distribution & $60(61.9 \%)$ & $43(57.3 \%)$ & $17(77.3 \%)$ & 0.134 \\
\hline
\end{tabular}

Data are $\mathrm{n}(\%)$, mean (SD) 
Table 3ロCT score findings

\begin{tabular}{|c|c|c|c|c|}
\hline Variables & Total(n=97) & Mild/Ordinary Group $\square n=75 \square$ & Severe/critical Group $(\mathrm{n}=22)$ & $P$ Value \\
\hline CT score & $10.6 \square 9.7 \square$ & $8.13 \square 8.3 \square$ & $18.8 \square 10.0 \square$ & $<0.001$ \\
\hline CT score group & & & & $<0.001$ \\
\hline 0 score & $25(25.8 \%)$ & $23(30.7 \%)$ & $2(9.1 \%)$ & \\
\hline 1】score & $30(30.9 \%)$ & $27(36.0 \%)$ & $3(13.6 \%)$ & \\
\hline $10 \square$ score & $18(18.6 \%)$ & $14(14.7 \%)$ & $4(18.2 \%)$ & \\
\hline $20 \square$ score & $24(24.7 \%)$ & $11(14.7 \%)$ & $13(59.1 \%)$ & \\
\hline
\end{tabular}

Data are n (\%), mean (SD)

Table 4aMultivariate analysis for different clinical types of COVID-19 Pneumonia

\begin{tabular}{lcccc}
\hline & B & $P$ Value & OR & OR 95\%CI \\
\hline & -19.405 & 0.000 & .000 & -- \\
& 2.072 & 0.004 & 7.939 & $1.956 \square 32.222$ \\
ies & 3.082 & 0.006 & 21.807 & $2.382 \square 199.648$ \\
on & -6.830 & 0.040 & .001 & $.000 \square .723$ \\
lellitus & 6.770 & 0.038 & 871.511 & $1.445 \square 525451.273$ \\
ıse & 9.329 & 0.016 & $11255.0935 .627 \square 22512589.750$ \\
l discomfort & 5.623 & 0.075 & 276.851 & $.573 \square 133746.965$ \\
pain/Diarrhea & 4.747 & 0.003 & 42.378 & $3.536 \square 507.813$ \\
re & 2.784 & 0.008 & 112.342 & $3.429 \square 3681.103$ \\
& .900 & 0.012 & 16.177 & $1.828 \square 143.171$ \\
& & & 2.459 & $1.065 \square 5.680$ \\
\hline
\end{tabular}

a. All variables in the model include age $\square$ sex $\square$ comorbidities $\square$ hypertension $\square$ diabetes mellitus $\square$ heart disease $\square$ pharyngeal discomfort $\square$ abdominal pain/Diarrhea[Temperature and CT score.

b. For Omnibus tests of model coefficients, $\chi 2=60.999, P<0.001$.

c. For model summary, Nagelkerke $\mathrm{R}^{2}=0.708$.

Figures 

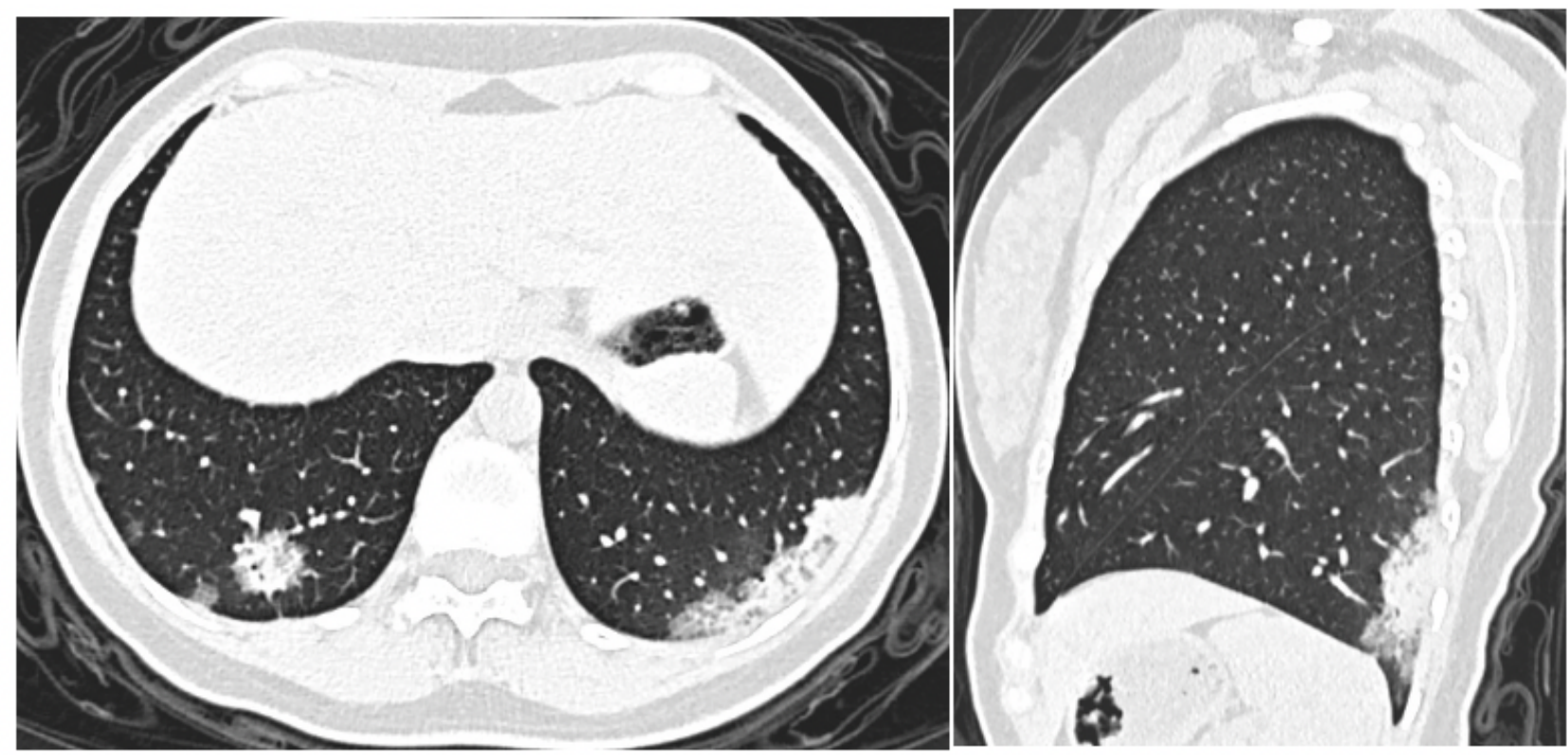

A.

B.

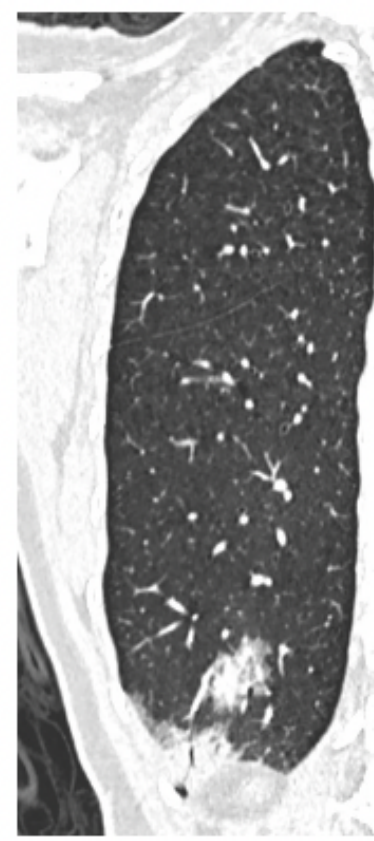

C.

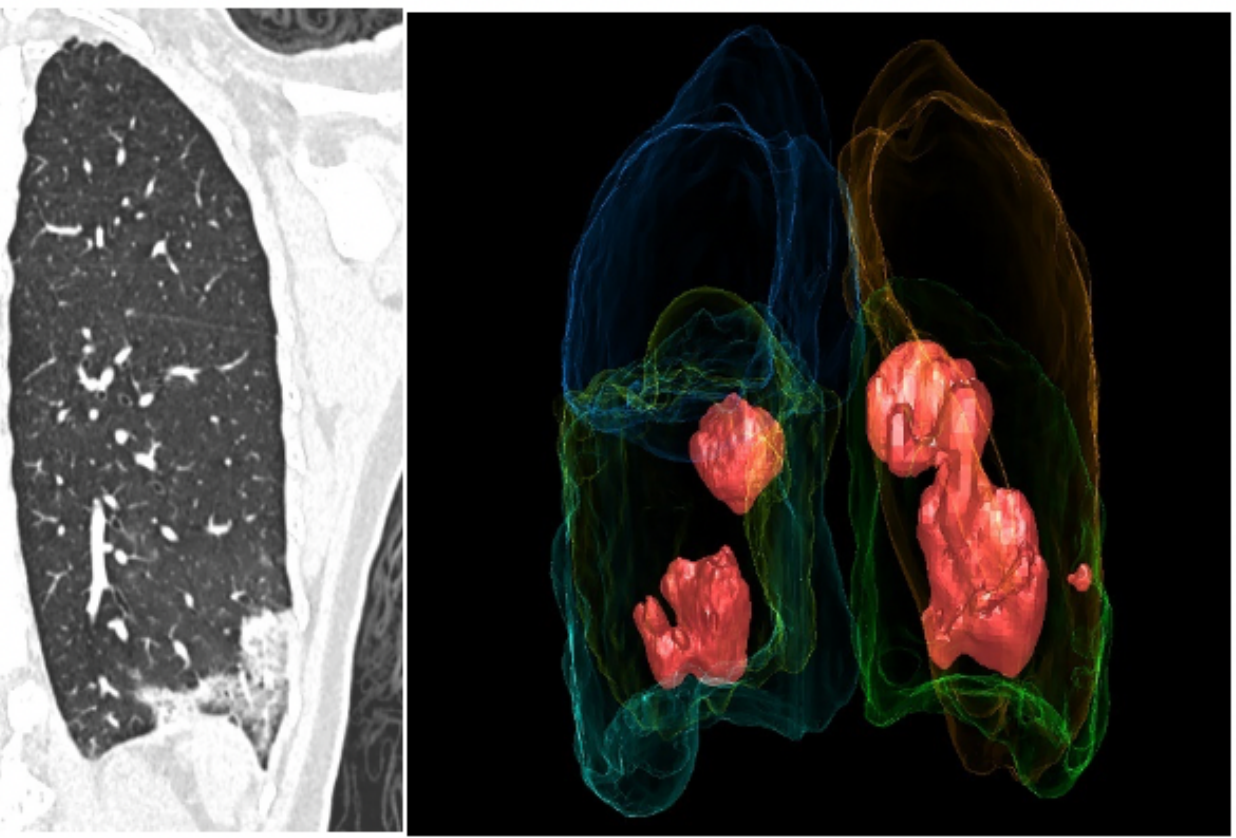

D.

\section{Figure 1}

Chest CT of a 33-year-old woman with ordinary COVID-19 pneumonia (CT score $=9$ ). (A-C) Axial, sagittal and coronal CT image showed small and large regions of subpleural GGO with irregular solid nodules. (B) 3D-visualization image showed the extent of GGO with scattered pattern. 

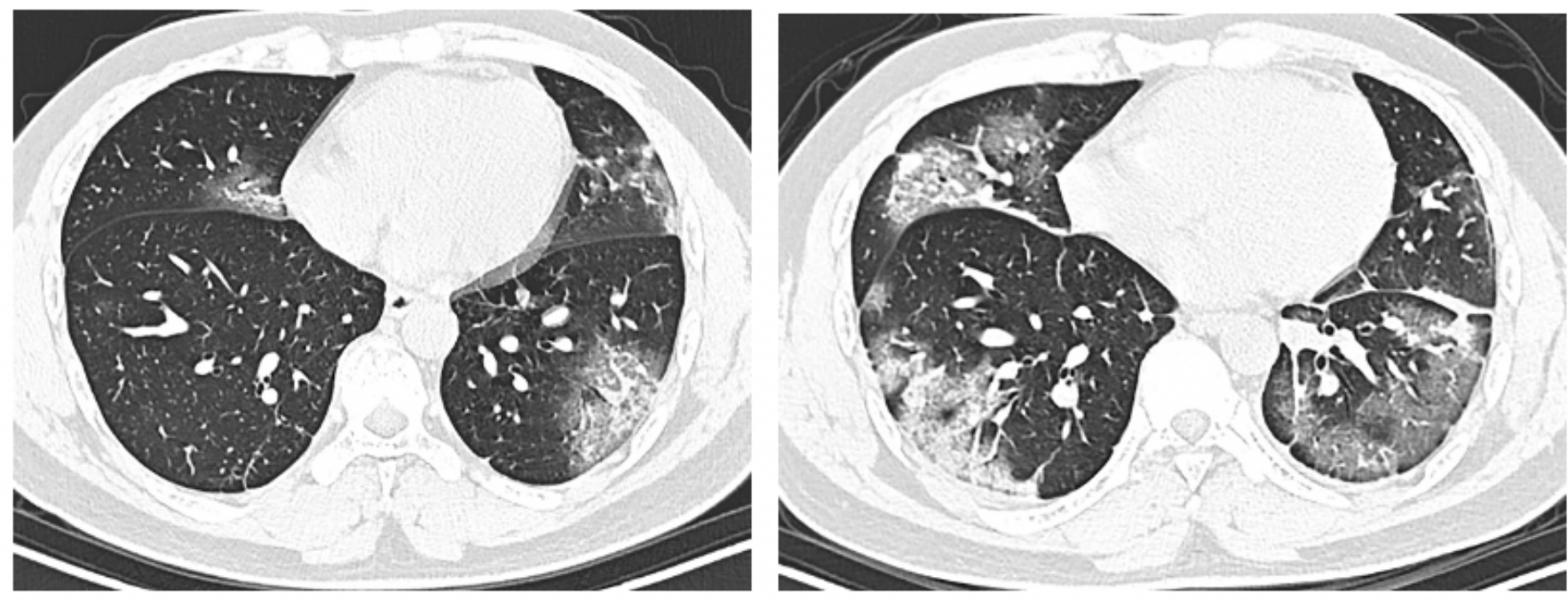

A.

B.
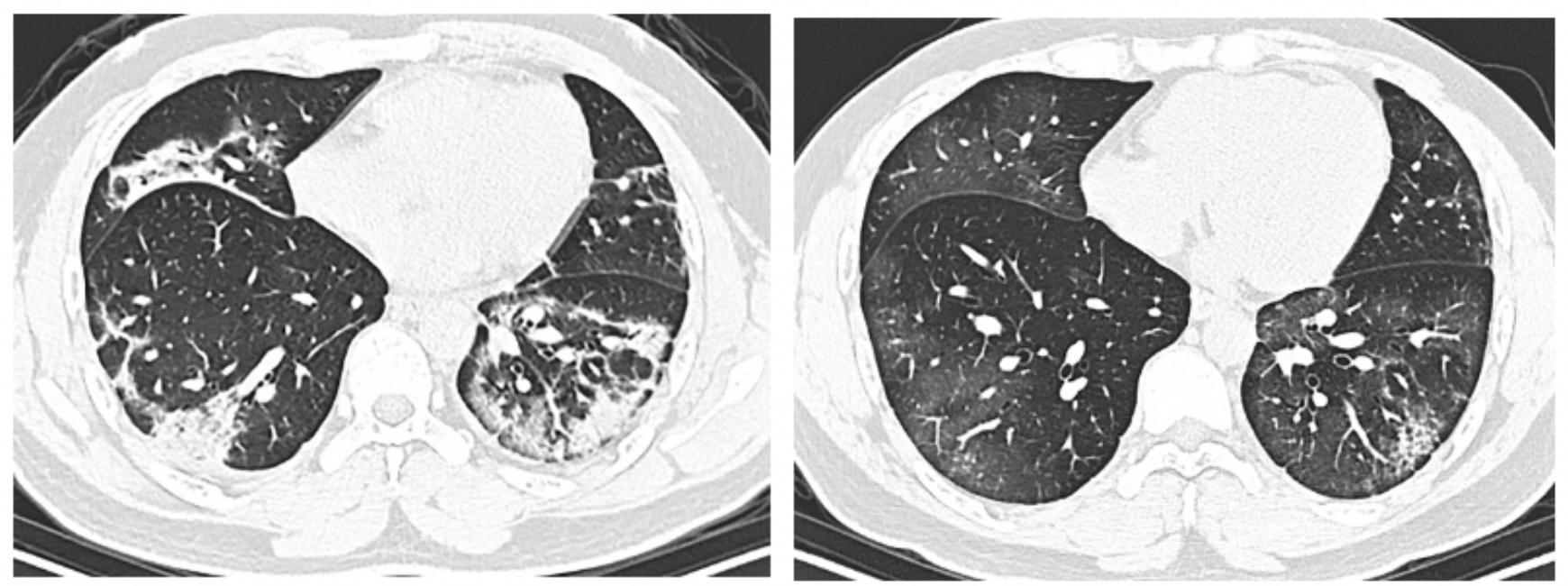

C.

D.

\section{Figure 2}

Chest CT of a 36-year-old man with ordinary COVID-19 pneumonia (CT score $=23$ ). A. Chest CT at admission showed multiple subpleural GGO lesions in the bilateral lung. B. 3 days later, the follow-up CT image showed the scope of the lesion began to expand and the density began to increase. C. 6 days later, the lesions increased further with superimposed inter- and intralobular septal thickening and consolidation. D. After 12 days, The density of the lesion is reduced. 